\title{
Changes in fatty acid compositions of total serum and lipoprotein particles, in growing rats given protein-deficient diets with either hydrogenated coconut or salmon oils as fat sources
}

\author{
BY MAHMOUD BOUZIANE, JOSIANE PROST AND JACQUES BELLEVILLE \\ Unité de Recherches de Nutrition Cellulaire et Métabolique, Université de Bourgogne, \\ Faculté des Sciences Mirande, BP 138, 21004 Dijon Cedex, France
}

(Received 25 September 1992 - Revised 24 May 1993 - Accepted 8 June 1993)

\begin{abstract}
The present study examines the effects of dietary saturated (hydrogenated coconut oil) and polyunsaturated (salmon oil) fats on the composition and metabolism of lipoproteins in growing rats fed on protein-deficient diets. Four groups of rats were fed on the following diets for $28 \mathrm{~d}: 200 \mathrm{~g}$ casein $+50 \mathrm{~g}$ coconut oil $(\mathrm{COC}) / \mathrm{kg}, 20 \mathrm{~g}$ casein $+50 \mathrm{~g}$ coconut oil $(\mathrm{COd}) / \mathrm{kg}, 200 \mathrm{~g}$ casein $+50 \mathrm{~g}$ salmon oil $(\mathrm{SAC}) / \mathrm{kg}, 20 \mathrm{~g}$ casein $+50 \mathrm{~g}$ salmon oil (SAd) $/ \mathrm{kg}$. Both protein-deficient groups exhibited low concentrations of protein and triacylglycerol (in serum, very-low-density lipoprotein (VLDL), lowdensity lipoprotein-high-density lipoprotein, (LDL-HDL $)_{1}$ and $H D L_{2-3}$ ), of cholesterol (in $L D L-H D L_{1}$ ) and of phospholipids (in VL.DL). Furthermore, serum and VLDL cholesterol concentrations were also reduced in the SAd group. Compared with rats given $200 \mathrm{~g}$ casein/kg diets, those fed on low-protein diets presented lower linoleic and arachidonic acid levels, in serum phospholipids and a dramatic decrease in the polyunsaturated:saturated fatty acid value. Relative amounts of linoleic and arachidonic acids in phospholipids of VLDL and $\mathrm{HDL}_{2-3}$ were also lowered in the COd group but not in the SAd group. However, proportions of 22:5n-6 and 22:6n-3 in VLDL and HDL $_{2-3}$ phospholipid fractions were enhanced in the COd and SAd groups respectively. The most affected apolipoproteins (apo) were apo $B_{100}$ and apo $B_{48}$ in rats fed on protein-deficient diets, apo $A_{I}$ and apo $E$ in the COd group, and apo $A_{I V}$ in the SAd group. Compared with rats fed hydrogenated coconut oil diets, those fed salmon oil diets had enhanced $L D L-H_{1}$ and $\mathrm{HDL}_{2-3}$ but lower VLDL total apolipoproteins (mainly due to a fall in apo $B_{100}$ and apo $B_{48}$ ). Arachidonic and eicosapentaenoic acids, which are impaired by protein deficiency, are the precursors of prostaglandins, thromboxanes and leukotrienes which are implicated in a number of regulatory processes. Our results demonstrate that protein malnutrition is associated with impaired metabolism of arachidonic and eicosapentaenoic acids. Protein malnutrition and essential fatty acid (EFA) deficiency are characterized by many common clinical features and the link between the two may be an impaired production of eicosanoids, since arachidonic and eicosapentaenoic acids are the precursors of these important metabolic regulators. Because of the apparent involvement of EFA deficiency in the aetiology of protein malnutrition, it may be prudent to include adequate amounts of EFA in diets of infants suffering from kwashiorkor.
\end{abstract}

Protein deficiency: EFA deficiency: Blood lipids: Salmon oil: Coconut oil: Rat

High intakes of saturated fatty acids (hydrogenated coconut oil) promote, in serum phospholipids, a pattern of polyunsaturated fatty acids (PUFA) characteristic of essential fatty acid (EFA) deficiency (Holman, 1960). Coconut oil, which is rich in lauric acid, raises serum triacylglycerol (TG) concentrations (Hostmark et al. 1982). Hill \& Holman (1980) have reported the effects of various dietary protein levels $(50-400 \mathrm{~g} / \mathrm{kg})$ on EFA availability in rats fed on hydrogenated coconut oil, and have indicated that signs observed in EFA deficiency are amplified with low protein intake. It has been proposed that EFA deficiency 
may contribute to a number of symptoms associated with kwashiorkor, e.g. fatty liver, scaly dermatitis with a tendency to superinfection, loss of hair, impaired wound healing and growth retardation, which are also observed in EFA deficiency alone (Koletzko et al. 1986).

On the other hand, PUFA affect lipid concentrations in plasma and lipoproteins. In fish oil the long-chain $n-3$ PUFA, namely eicosapentaenoic acid $(20: 5 n-3$; EPA) and docosahexaenoic acid (22:6n-3; DHA) are the active components in lowering plasma lipids. EPA and arachidonic acid serve as substrates for cyclooxygenases and lipoxygenases, the enzymes that initiate the synthesis of prostaglandins, thromboxanes, prostacyclins and leukotrienes. Fish oils (e.g. salmon oil) are known to affect platelet function by reducing platelet aggregation and adhesion (Hornstra, 1989), and plasma lipids by reducing plasma concentration of very-low-density lipoprotein (VLDL) and, thereby, plasma TG levels (Harris et al. 1983).

Protein deficiency, which affects the synthesis of enzymes involved in lipid metabolism (Narce et al. 1988), and apolipoprotein (apo) compositions (Meghelli-Bouchenak et al. 1989 a), may be a component in the adverse effect on PUFA status in some human diseases (Holman, 1986). Thus, results obtained in those studies indicate relationships between protein intake and EFA utilization and metabolism. In addition to decreased intake and synthesis, the increased oxidative metabolism of PUFA may accelerate marginal EFA deficiency and decrease long-chain PUFA bioavailability, thus increasing EFA requirements. However, there is only scant information on the supply and metabolism of EFA in malnourished children. Food intakes of infants before their hospital admission are difficult to determine and the results of several studies were inconsistent (see Dhansay et al. 1991). Similarly, little attention has been accorded to PUFA supplies in protein-deficiency studies in animals. Variations may also be explained by the different degree and duration of protein malnutrition.

In the present study in the growing rat, EFA, other long-chain PUFA, and protein intakes were determined exactly. Diets were provided for a short period (28 d). The combined influence of dietary protein levels (200 or $20 \mathrm{~g}$ casein $/ \mathrm{kg}$ ) associated with saturated (coconut oil) or polyunsaturated (salmon oil) fatty acids was determined in serum and lipoprotein fractions to measure their capacity to carry PUFA.

\section{MATERIALS AND METHODS}

\section{Animals and diets}

Forty male Wistar rats (Iffa Credo, l'Arbresle, Lyon, France) weighing 80 (SD 6) g at the beginning of the experiment were allowed free access to an adequate diet (containing $200 \mathrm{~g}$ casein and $50 \mathrm{~g}$ olive oil $/ \mathrm{kg}$ ) for $10 \mathrm{~d}$. After this adaptation period, when their body weight was 112 (SD 8) g, they were randomly divided into four groups. For $28 \mathrm{~d}$, two groups (controls) were fed on adequate diets containing $200 \mathrm{~g}$ casein $/ \mathrm{kg}$ and either $50 \mathrm{~g}$ hydrogenated coconut oil $/ \mathrm{kg}$ (rich in saturated fatty acids; COC), or $50 \mathrm{~g}$ salmon oil (rich in EPA and DHA; SAC). Two other groups were given low-protein diets $(20 \mathrm{~g}$ casein $/ \mathrm{kg})$ and either $50 \mathrm{~g}$ coconut oil $/ \mathrm{kg}(\mathrm{COd})$, or $50 \mathrm{~g}$ salmon oil $/ \mathrm{kg}$ (SAd).

The coconut oil composition (g/100 g total fatty acids) was as follows: $8: 04 \cdot 0,10: 060$, 12:0 39.0, 14:0 19.0, 16:0 16.0, 18:0 4.6, 18:1 (n-9+n-7) 8.9, 18:2n-6 1.6. The salmon oil composition was as follows: $14: 02 \cdot 0,16: 016 \cdot 0,16: 1 n-76 \cdot 0,18: 04 \cdot 0,18: 1(n-9+n-7) 18 \cdot 0$, $18: 2 n-64 \cdot 0,20: 1(n-9+n-11) 10 \cdot 0,20: 4 n-61 \cdot 1,20: 5 n-39 \cdot 9,22: 1(n-9+n-11) 8 \cdot 0,22: 5 n-3$ $3 \cdot 9,22: 6 n-311 \cdot 1,24: 1 n-91 \cdot 2$. The composition of the diets is shown in Table 1 .

Diets were isoenergetic $(16.28 \mathrm{MJ} / \mathrm{kg})$ and contained the same quantities of lipids, vitamins, minerals and fibre. Animals were maintained in wire-bottomed cages at constant temperature $\left(24^{\circ}\right)$ and humidity $(60 \%)$ with a $12 \mathrm{~h}$ light cycle $(07.00-19.00$ hours). They ate 
Table 1. Diet compositions $(\mathrm{g} / \mathrm{kg}$ diet $)$

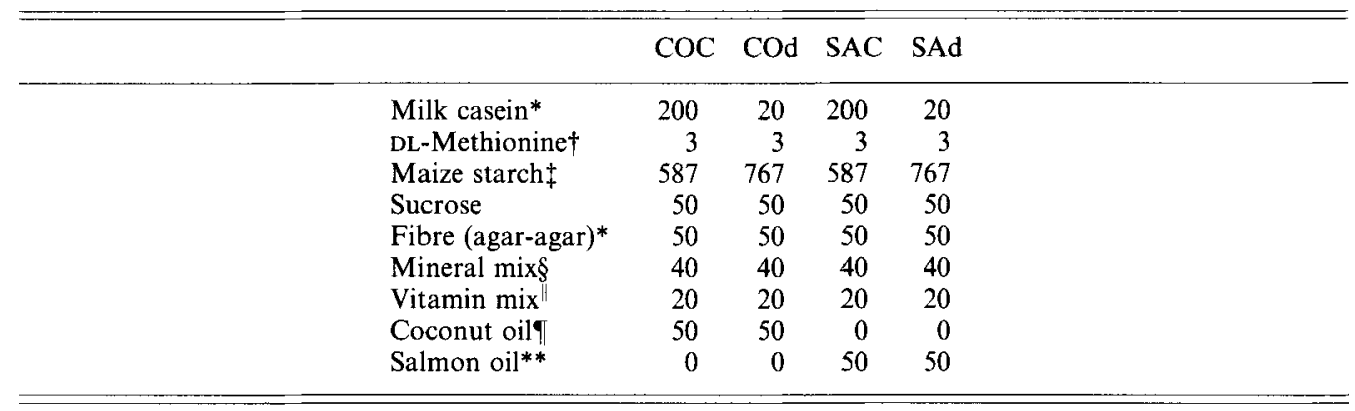

The diets were isoenergetic $(16.80 \mathrm{MJ} / \mathrm{kg})$ and given in powdered form.

* Prolabo, Paris, France.

$\dagger$ Merck, Darmstadt, Germany.

$\ddagger$ Ets Louis François, Saint Maur les Fossés, France.

$\$$ UAR 205 B (Villemoisson, 91360 Epinay/Orge, France). Mineral mix provided the following ( $\mathrm{g} / \mathrm{kg}$ diet): Ca 4, K 2.4, Na 1.6, Mg 0.4, Fe 0.12, Mn 0.032, Cu 0.005, Zn 0.018, Co 0.00004, I 0.00002.

I UAR 200 (Villemoisson). Vitamin mix provided the following ( $\mathrm{mg} / \mathrm{kg} \mathrm{diet}$ ): thiamin 40, riboflavin 30 , nicotinic acid 140, pyridoxine 20, pyridoxal 300 , cyanocobalamin $0 \cdot 1$, ascorbic acid 1600 , $\alpha$-tocopherol 340 , menadione 80 , calcium pantothenate 200 , choline 2720 , pteroylmonoglutamic acid $10, p$-aminobenzoic acid 100 , biotin 0.6, retinol 12, cholecalciferol $0 \cdot 125$.

T Astra Calvé, Asniere, France.

** Gattefossé, St Priest, France.

and drank ad lib. We followed the general guidelines for the care and use of laboratory animals (Council of European Communities, 1986).

\section{Blood samples}

On day 28 of the experiment, after an overnight fast, rats were anaesthetized with sodium pentobarbital ( $60 \mathrm{mg} / \mathrm{kg}$ body weight) before blood collection by abdominal puncture. Serum was obtained by low-speed centrifugation and preserved with $0.26 \mathrm{mmol}$ $\mathrm{Na}_{2}$-EDTA/l and $3 \mathrm{mmol} \mathrm{NaN}_{3} / \mathrm{l}$.

Total lipoprotein (density $<1.21 \mathrm{~kg} / \mathrm{l}$ ) isolation. The density of the serum was adjusted to $1.21 \mathrm{~kg} / \mathrm{l}$ by addition of crystalline $\mathrm{KBr}(0.322 \mathrm{~kg} / 1$; Havel et al. 1955). Lipoproteins of density $(d)<1.21 \mathrm{~kg} / 1$ were isolated from about $2 \mathrm{ml}$ of each serum sample by a single centrifugation flotation (Model L 8-55 ultracentrifuge, $50 \mathrm{Ti}$ rotor; Beckman Instruments, Palo Alto, CA, USA). All centrifugations were performed at $122000 \mathrm{~g}$ at $15^{\circ}$ for $48 \mathrm{~h}$. Lipoproteins were dialysed against $0.15 \mathrm{M} \mathrm{NaCl}$ and $1 \mathrm{mM} \mathrm{Na}_{2}$ EDTA, pH 7.4 at $4^{\circ}$ in Spectra/Por 2 dialysis tubing (Spectrum Medical Industries, Los Angeles, CA, USA).

Isolation of lipoprotein fractions. Three fractions (VLDL, low-density-lipoprotein-highdensity-lipoprotein $\left(\mathrm{LDL}-\mathrm{HDL}_{1}\right), \mathrm{HDL}_{2-3}$ ) were isolated by a single-spin discontinuous gradient (Redgrave et al. 1975, modified by Meghelli-Bouchenak et al. 1989a). Each discontinuous gradient was constructed at ambient temperature in a thin-walled polyallomer tube $(13.5 \mathrm{~mm}$ diameter $\times 89 \mathrm{~mm}$ length) of the Beckman SW41-Ti swinging bucket rotor. The centrifugation was performed at $274000 \mathrm{~g}$ at $15^{\circ}$ in a Beckman L5-50 ultracentrifuge. VLDL portions $(2 \mathrm{ml})$ were taken with a syringe at the top of the tube. Then the content of the tube was displaced by a KBr solution of $d 1.346 \mathrm{~kg} / \mathrm{l}$ at the bottom of the tube and collected from the surface. During the displacement, this effluent was monitored by measurement of absorption at $280 \mathrm{~nm}$ in a Beckman spectrophotometer (Model 35) to isolate the LDL- $\mathrm{HDL}_{1}$ and $\mathrm{HDL}_{2-3}$ peaks. 
Table 2. The effects of control $(C)$ or protein-deficient $(d)$ diets containing coconut $(C O)$ or salmon oil $(S A)$ on protein $(g / l)$ and lipid contents $(\mathrm{mmol} / \mathrm{l})$ of serum, very-low-density lipoprotein $(V L D L)$, low-density lipoprotein-high-density lipoprotein $\left(L D L-H D L_{1}\right)$ and $H D L_{2-3}$ fractions of growing rats*

(Values are means for six rats with their pooled standard errors)

\begin{tabular}{|c|c|c|c|c|c|c|c|c|}
\hline \multirow[b]{2}{*}{ Diet... } & \multirow[b]{2}{*}{$\mathrm{COC}$} & \multirow[b]{2}{*}{ COd } & \multirow[b]{2}{*}{ SAC } & \multirow[b]{2}{*}{ SAd } & \multirow[b]{2}{*}{ SEM } & \multicolumn{3}{|c|}{$\begin{array}{c}\text { Statistical significance }(P) \\
\text { of effect of }\end{array}$} \\
\hline & & & & & & $\begin{array}{c}\text { oil type } \\
\text { (CO v. SA) }\end{array}$ & $\begin{array}{l}\text { level of } \\
\text { protein } \\
\text { (C v.d) }\end{array}$ & $\begin{array}{l}\text { Interaction } \\
\quad \text { (oil } \\
\text { type } \times \text { level } \\
\text { of protein) }\end{array}$ \\
\hline \multicolumn{9}{|l|}{ Protein } \\
\hline :Serum & $58 \cdot 9$ & 45.8 & $68 \cdot 9$ & 47.7 & 1.76 & 0.003 & $<0.001$ & 0.032 \\
\hline VLDL & 0.26 & 0.17 & 0.21 & 0.14 & 0.02 & 0.059 & $<0.001$ & $<0.622$ \\
\hline LDL-HDL & 0.08 & 0.06 & $0 \cdot 15$ & $0 \cdot 10$ & 0.05 & $<0.001$ & $<0.001$ & 0.007 \\
\hline $\mathrm{HDL}_{2-3}$ & $0 \cdot 71$ & 0.56 & 1.05 & 0.74 & 0.06 & $<0.001$ & $<0.001$ & 0.554 \\
\hline \multicolumn{9}{|l|}{ Phospholipids } \\
\hline :Serum & $44 \cdot 8$ & $43 \cdot 9$ & $35 \cdot 2$ & $33 \cdot 9$ & 2.78 & $0 \cdot 002$ & 0.693 & $>0.90$ \\
\hline VLDL & $5 \cdot 36$ & 3.06 & $3 \cdot 77$ & 3.06 & $0 \cdot 38$ & 0.049 & $<0.001$ & 0.049 \\
\hline LDL-HDL ${ }_{1}$ & $4 \cdot 84$ & $4 \cdot 84$ & $5 \cdot 49$ & $5 \cdot 81$ & 0.59 & $0 \cdot 185$ & $0 \cdot 790$ & 0.790 \\
\hline $\mathrm{HDL}_{2-3}$ & $17 \cdot 3$ & $21 \cdot 8$ & 16.8 & $24 \cdot 5$ & 1.08 & $0 \cdot 319$ & $<0.001$ & 0.154 \\
\hline \multicolumn{9}{|l|}{ Triacylglycerols } \\
\hline :Serum & 1.65 & 0.89 & $1 \cdot 11$ & 0.49 & 0.05 & $<0.001$ & $<0.001$ & $0 \cdot 176$ \\
\hline VLDL & $1 \cdot 14$ & $0 \cdot 60$ & 0.50 & 0.24 & 0.05 & $<0.001$ & $<0.001$ & 0.011 \\
\hline $\mathrm{LDL}_{-} \mathrm{HDL}_{1}$ & nd & nd & nd & nd & - & - & - & - \\
\hline $\mathrm{HDL}_{2-3}$ & nd & nd & nd & nd & - & - & - & - \\
\hline \multicolumn{9}{|c|}{ Unesterified cholesterol } \\
\hline :Serum & 0.68 & 0.70 & $0 \cdot 50$ & 0.48 & 0.06 & 0.003 & $>0.99$ & 0.744 \\
\hline VLDL & $0 \cdot 14$ & 0.19 & 0.07 & 0.02 & 0.01 & $<0.001$ & $>0.99$ & $<0.001$ \\
\hline $\mathrm{LDL}-\mathrm{HDL}_{1}$ & $0 \cdot 16$ & 0.08 & 0.26 & 0.04 & 0.02 & $0 \cdot 149$ & $<0.001$ & 0.002 \\
\hline $\mathrm{HDL}_{2-3}$ & 0.37 & 0.48 & 0.32 & 0.38 & 0.03 & 0.021 & $0 \cdot 010$ & 0.416 \\
\hline \multicolumn{9}{|c|}{ Esterified cholesterol } \\
\hline :Serum & $2 \cdot 85$ & $2 \cdot 80$ & $2 \cdot 02$ & 1.61 & $0 \cdot 11$ & $<0.001$ & 0.049 & $0 \cdot 118$ \\
\hline VLDL & 0.35 & 0.38 & 0.37 & 0.13 & 0.04 & 0.009 & $0 \cdot 016$ & 0.003 \\
\hline $\mathrm{LDL}_{-} \mathrm{HDL}_{1}$ & 0.28 & 0.09 & 0.34 & $0 \cdot 19$ & 0.03 & 0.004 & $<0.001$ & $<0.001$ \\
\hline $\mathrm{HDL}_{2-3}$ & 1.41 & 0.68 & 0.77 & 0.64 & 0.05 & $<0.001$ & $<0.001$ & $<0.001$ \\
\hline \multicolumn{9}{|l|}{ Total cholesterol } \\
\hline :Serum & 3.55 & $3 \cdot 51$ & $2 \cdot 54$ & $2 \cdot 10$ & $0 \cdot 23$ & $<0.001$ & $0 \cdot 310$ & 0.394 \\
\hline VLDL & $0 \cdot 50$ & 0.58 & $0 \cdot 44$ & $0 \cdot 16$ & 0.05 & $<0.001$ & 0.059 & 0.001 \\
\hline $\mathrm{LDL}_{-} \mathrm{HDL}_{1}$ & 0.44 & $0 \cdot 17$ & 0.60 & 0.23 & 0.04 & 0.012 & $<0.001$ & 0.225 \\
\hline $\mathrm{HDL}_{2-3}$ & 1.78 & $1 \cdot 17$ & $1 \cdot 10$ & 1.02 & 0.07 & $<0.001$ & $<0.001$ & 0.001 \\
\hline
\end{tabular}

COC, $200 \mathrm{~g}$ casein $+50 \mathrm{~g} \mathrm{CO} / \mathrm{kg}$; COd, $20 \mathrm{~g}$ casein $+50 \mathrm{~g} \mathrm{CO} / \mathrm{kg} ; \mathrm{SAC}, 200 \mathrm{~g}$ casein $+50 \mathrm{~g} \mathrm{SA} / \mathrm{kg} ; \mathrm{SAd}, 20 \mathrm{~g}$ casein $+50 \mathrm{~g} \mathrm{SA} / \mathrm{kg}$; nd, not determined.

* For details of diets and procedures, see Table 1 and pp. 376-379.

Purified fractions of VLDL $(d<1.006)$, LDL-HDL 1 (1.006 $<d<1.06)$ and $\mathrm{HDL}_{2-3}$ $(1.06<d<1 \cdot 15)$ were dialysed as described previously.

\section{Chemical analyses}

Liver, serum and lipoprotein total lipids were extracted according to the method of Folch et al. (1957). The amount of total lipid per $\mathrm{g}$ liver was determined gravimetrically from a subsample. Phospholipid (PL) and TG fractions were isolated by TLC (Stahl et al. 1956). PL and TG fractions were methylated, and then fatty acid analysis of PL and TG fractions 
Table 3. The effects of control $(C)$ or protein-deficient $(d)$ diets containing coconut $(C O)$ or salmon oil $(S A)$ on major fatty acid compositions of serum triacylglycerols $(\mathrm{mg} / 100 \mathrm{mg}$ fatty acids) of growing rats*

(Values are means for six rats with their pooled standard errors)

\begin{tabular}{|c|c|c|c|c|c|c|c|c|}
\hline \multirow[b]{2}{*}{ Diet... } & \multirow[b]{2}{*}{$\mathrm{COC}$} & \multirow[b]{2}{*}{ COd } & \multirow[b]{2}{*}{ SAC } & \multirow[b]{2}{*}{ SAd } & \multirow[b]{2}{*}{ SEM } & \multicolumn{3}{|c|}{$\begin{array}{c}\text { Statistical significance }(P) \\
\text { of effect of }\end{array}$} \\
\hline & & & & & & $\begin{array}{c}\text { oil type } \\
\text { (CO v. SA) }\end{array}$ & $\begin{array}{l}\text { level of } \\
\text { protein } \\
(\mathrm{C} v . \mathrm{d})\end{array}$ & $\begin{array}{c}\text { Interaction } \\
\text { (oil type } \times \text { level } \\
\text { of protein) }\end{array}$ \\
\hline Total saturated & $32 \cdot 5$ & $42 \cdot 3$ & $22 \cdot 0$ & $37 \cdot 0$ & $2 \cdot 85$ & 0.012 & $<0.001$ & $0 \cdot 374$ \\
\hline Total monounsaturated & $54 \cdot 4$ & $47 \cdot 4$ & 27.9 & $24 \cdot 4$ & 3.42 & $<0.001$ & $0 \cdot 139$ & $0 \cdot 615$ \\
\hline $20: 3 n-9$ & 1.9 & $2 \cdot 9$ & 0.9 & 1.0 & $0 \cdot 28$ & $<0.001$ & 0.064 & $0 \cdot 124$ \\
\hline $18: 2 n-6$ & $5 \cdot 8$ & $3 \cdot 0$ & $5 \cdot 7$ & $5 \cdot 8$ & 0.44 & $<0.001$ & $<0.001$ & 0.003 \\
\hline $20: 4 n-6$ & $3 \cdot 0$ & $2 \cdot 1$ & 0.7 & 1.9 & 0.29 & $<0.001$ & 0.616 & 0.001 \\
\hline $22: 5 n-6$ & 0.8 & $1 \cdot 8$ & 0.6 & $1 \cdot 2$ & 0.23 & 0.098 & 0.001 & $0 \cdot 396$ \\
\hline Total $n-6$ & $9 \cdot 6$ & $6 \cdot 9$ & $7 \cdot 0$ & 8.9 & 0.89 & 0.738 & 0.658 & 0.018 \\
\hline $18: 3 n-3$ & nd & nd & 1.6 & 0.5 & 0.07 & $\ldots$ & $<0.001$ & - \\
\hline $20: 5 n-3$ & $\operatorname{tr}$ & tr & $18 \cdot 3$ & 5.9 & $1 \cdot 82$ & - & $<0.001$ & - \\
\hline $22: 5 n-3$ & nd & nd & $3 \cdot 9$ & $3 \cdot 2$ & 0.55 & - & $0 \cdot 270$ & - \\
\hline $22: 6 n-3$ & $\operatorname{tr}$ & $\operatorname{tr}$ & 17.9 & 18.8 & 1.91 & - & 0.720 & - \\
\hline Total $n-3$ & $\mathrm{tr}$ & $\operatorname{tr}$ & $41 \cdot 7$ & $28 \cdot 4$ & $4 \cdot 12$ & - & 0.009 & - \\
\hline$P: S$ & $2 \cdot 02$ & $1 \cdot 35$ & $3 \cdot 52$ & 1.69 & $0 \cdot 29$ & 0.004 & $<0.001$ & 0.064 \\
\hline $20: 4 n-6 / 18: 2 n-6$ & 0.51 & $0 \cdot 70$ & $0 \cdot 12$ & $0 \cdot 32$ & 0.04 & $<0.001$ & $<0.001$ & 0.90 \\
\hline $20: 3 n-9 / 20: 4 n-6$ & 0.63 & $1 \cdot 38$ & $1 \cdot 28$ & 0.52 & 0.07 & $0 \cdot 15$ & $>0.90$ & $<0.001$ \\
\hline
\end{tabular}

COC, $200 \mathrm{~g}$ casein $+50 \mathrm{~g} \mathrm{CO} / \mathrm{kg} ; \mathrm{COd}, 20 \mathrm{~g}$ casein $+50 \mathrm{~g} \mathrm{CO} / \mathrm{kg} ; \mathrm{SAC}, 200 \mathrm{~g}$ casein $+50 \mathrm{~g} \mathrm{SA} / \mathrm{kg} ; \mathrm{SAd}, 20 \mathrm{~g}$ casein $+50 \mathrm{~g} \mathrm{SA} / \mathrm{kg}$; nd, not detected; tr, trace $(<0 \cdot 1) ; \mathrm{P}: \mathrm{S}$, polyunsaturated: saturated.

* For details of diets and procedures, see Table 1 and pp. 376-379.

was performed by GLC (Slover \& Lanza, 1979), using a Becker Gas Chromatograph Packard 417, equipped with a glass column, length $39 \mathrm{~m}$, internal diameter $0.3 \mathrm{~mm}$, stationary phase carbowax $20 \mathrm{M}, \mathrm{H}_{2}$ flow-rate $6 \mathrm{ml} / \mathrm{min}$, inlet heater $202^{\circ}$, detector temperature $240^{\circ}$ (Becker Instruments, Downers Grove, IL, USA), with heptadecanoic acid as internal standard. Identification of fatty acids was performed with commercial standards by means of relative retention times. Areas were calculated with an ENICA 21 integrator (DELSI Instrument, Suresnes, France). The average molecular weight of fatty acids was determined, allowing amounts of serum and VLDL TG to be calculated. The solutions used for lipid extraction and TLC contained 2,6 di-tert-butyl-p-cresol $(50 \mathrm{mg} / 1)$ as an antioxidant, and the lipid extracts were stored under $\mathrm{N}_{2}$ gas in the dark at $-20^{\circ}$ to prevent peroxidation of unsaturated fatty acids.

Total cholesterol (TC) determination was performed by GLC (Gambert et al. 1979) and unesterified cholesterol was measured according to the method of Gambert et al. (1982). PL were quantified by the measurement of $\mathrm{P}$ (Bartlett, 1958). Protein contents were measured (Lowry et al. 1951) using bovine serum albumin as standard.

\section{Electrophoretic evaluation of VLDL and HDL apolipoproteins}

After partial delipidation, VLDL and $\mathrm{HDL}_{2-3}$ apolipoproteins were estimated using SDS-PAGE $(2 \cdot 5>20 \%)$ by the method of Meghelli-Bouchenak et al. (1989a). Electrophoresis was performed in a LKB 2001-001 vertical electrophoresis unit (LKB Produkte, Bromme, Sweden) at $4^{\circ}$, for $18 \mathrm{~h}$ with $20 \mathrm{~mA} /$ gel slab. Gels were then stained with Coomassie brilliant blue $\mathrm{G} 250$. 
Table 4. The effects of control $(C)$ or protein-deficient $(d)$ diets containing coconut $(C O)$ or salmon oil $(S A)$ on major fatty acid compositions of very-low-density lipoprotein (VLDL) triacylglycerols ( $\mathrm{mg} / 100 \mathrm{mg}$ fatty acids) of growing rats*

(Values are means for six rats with their pooled standard errors)

\begin{tabular}{|c|c|c|c|c|c|c|c|c|}
\hline \multirow[b]{2}{*}{ Diet... } & \multirow[b]{2}{*}{$\mathrm{COC}$} & \multirow[b]{2}{*}{ cod } & \multirow[b]{2}{*}{ SAC } & \multirow[b]{2}{*}{ SAd } & \multirow[b]{2}{*}{ SEM } & \multicolumn{3}{|c|}{$\begin{array}{c}\text { Statistical significance }(P) \\
\text { of effect of }\end{array}$} \\
\hline & & & & & & $\begin{array}{c}\text { oil type } \\
(\mathrm{CO} \text { v. SA) }\end{array}$ & $\begin{array}{l}\text { level of } \\
\text { protein } \\
(\mathrm{C} v . \mathrm{d})\end{array}$ & $\begin{array}{c}\text { Interaction } \\
\text { (oil type } \times \text { level } \\
\text { of protein) }\end{array}$ \\
\hline Total saturated & $44 \cdot 6$ & $55 \cdot 6$ & $34 \cdot 0$ & $43 \cdot 2$ & $2 \cdot 70$ & $<0.001$ & $<0.001$ & 0.746 \\
\hline Total monounsaturated & $43 \cdot 6$ & $35 \cdot 2$ & $23 \cdot 3$ & $21 \cdot 9$ & $2 \cdot 82$ & $<0 \cdot 001$ & 0.098 & $0 \cdot 23$ \\
\hline $20: 3 n-9$ & 0.9 & $1 \cdot 2$ & 0.6 & 0.9 & $0 \cdot 23$ & 0.208 & $0 \cdot 208$ & $>0.99$ \\
\hline $18: 2 n-6$ & $5 \cdot 2$ & $2 \cdot 5$ & $2 \cdot 4$ & $2 \cdot 2$ & $0 \cdot 38$ & $<0.001$ & $<0.001$ & $0 \cdot 003$ \\
\hline $20: 4 n-6$ & $4 \cdot 2$ & $2 \cdot 6$ & $2 \cdot 7$ & $2 \cdot 6$ & $0 \cdot 30$ & 0.242 & 0.010 & 0.212 \\
\hline $22: 5 n-6$ & 0.9 & $2 \cdot 0$ & nd & nd & $0 \cdot 29$ & - & 0.003 & - \\
\hline Total $n-6$ & $10 \cdot 3$ & $7 \cdot 1$ & $5 \cdot 1$ & $4 \cdot 8$ & $0-81$ & $<0.001$ & 0.043 & 0.088 \\
\hline $18: 3 n-3$ & nd & nd & $2 \cdot 5$ & $1 \cdot 0$ & 0.22 & - & $<0.001$ & - \\
\hline $20: 5 n-3$ & nd & nd & $15 \cdot 2$ & $10 \cdot 4$ & $1 \cdot 10$ & - & 0.001 & - \\
\hline $22: 5 n-3$ & nd & nd & $4 \cdot 8$ & $2 \cdot 7$ & $0 \cdot 35$ & - & $<0.001$ & - \\
\hline $22: 6 n-3$ & nd & nd & $12 \cdot 9$ & $15 \cdot 0$ & 0.76 & - & 0.020 & - \\
\hline Total $n-3$ & nd & nd & $35 \cdot 4$ & $29 \cdot 1$ & $2 \cdot 40$ & - & 0.025 & - \\
\hline$P: S$ & $1 \cdot 22$ & $0 \cdot 78$ & 1.89 & $1 \cdot 31$ & $0 \cdot 15$ & $<0.001$ & $<0.001$ & 0.65 \\
\hline $20: 4 n-6 / 18: 2 n-6$ & 0.80 & 1.04 & $1 \cdot 12$ & $1 \cdot 18$ & 0.07 & $<0.001$ & 0.044 & $0 \cdot 215$ \\
\hline $20: 3 n-9 / 20: 4 n-6$ & $0 \cdot 21$ & $0 \cdot 46$ & $0 \cdot 22$ & 0.34 & 0.05 & $0-284$ & 0.001 & 0.208 \\
\hline
\end{tabular}

COC, $200 \mathrm{~g}$ casein $+50 \mathrm{~g} \mathrm{CO} / \mathrm{kg} ; \mathrm{COd}, 20 \mathrm{~g}$ casein $+50 \mathrm{~g} \mathrm{CO} / \mathrm{kg} ; \mathrm{SAC}, 200 \mathrm{~g}$ casein $+50 \mathrm{~g} \mathrm{SA} / \mathrm{kg} ; \mathrm{SAd}, 20 \mathrm{~g}$ casein $+50 \mathrm{~g} \mathrm{SA} / \mathrm{kg}$; nd, not detected; P:S, polyunsaturated:saturated.

* For details of diets and procedures, see Table 1 and pp. 376-379.

Destained gels were scanned at $600 \mathrm{~nm}$ with a densitometer (Model Profil 26; Sebia, Issy les Moulineaux, France). Apolipoproteins were determined semiquantitatively with the densitometer tracings. To estimate the concentration of each apolipoprotein, the percentage of the area relative to each apolipoprotein was multiplied by the total apolipoprotein content of each serum sample. Staining affinity of each peptide was not determined individually; however, when $50-200 \mathrm{mg}$ total protein was applied, the chromogenicity of each major band varied linearly with the amount of total protein applied to the gel. Apolipoprotein samples for the four groups were subjected to electrophoresis in parallel for each lipoprotein fraction. Results were expressed as arbitrary units (AU).

\section{Statistical analysis}

Results were reported as arithmetical means for each group with their pooled standard errors (SEM). The significance of differences were determined by Student's $t$ test for unpaired values. The differences were considered significant at $P<0.05$.

\section{RESULTS}

Body weights and food intakes

After $28 \mathrm{~d}$ on a protein-deficient diet, rats developed fatty livers. The total lipid contents (mg/g liver) were: 145 (SEM 13), 104 (SEM 11), 68 (SEM 1) and 58 (SEM 4), for the COd, SAd, COC and SAC groups respectively. The values for the protein-deficient groups were significantly greater than those for protein-sufficient groups $(P<0 \cdot 001)$. In spite of similar 
Table 5. The effects of control $(C)$ or protein-deficient $(d)$ diets containing coconut $(C O)$ or salmon oil $(S A)$ on major fatty acid compositions of serum phospholipids ( $\mathrm{mg} / 100 \mathrm{mg}$ fatty acids) of growing rats*

(Values are means for six rats with their pooled standard errors)

\begin{tabular}{|c|c|c|c|c|c|c|c|c|}
\hline \multirow[b]{2}{*}{ Diet ... } & \multirow[b]{2}{*}{$\mathrm{COC}$} & \multirow[b]{2}{*}{ cod } & \multirow[b]{2}{*}{$\mathrm{SAC}$} & \multirow[b]{2}{*}{ SAd } & \multirow[b]{2}{*}{ SEM } & \multicolumn{3}{|c|}{$\begin{array}{c}\text { Statistical significance }(P) \\
\text { of effect of }\end{array}$} \\
\hline & & & & & & $\begin{array}{c}\text { oil type } \\
\text { (CO v. SA) }\end{array}$ & $\begin{array}{l}\text { level of } \\
\text { protein } \\
(C \text { v. d) }\end{array}$ & $\begin{array}{c}\text { Interaction } \\
\text { (oil type } \times \text { level } \\
\text { of protein) }\end{array}$ \\
\hline Total saturated & $48 \cdot 7$ & $67 \cdot 3$ & $26 \cdot 7$ & $49 \cdot 1$ & $3 \cdot 24$ & $<0.001$ & $<0.001$ & 0.561 \\
\hline Total monounsaturated & $30 \cdot 3$ & $17 \cdot 2$ & $18 \cdot 9$ & $17 \cdot 2$ & $2 \cdot 30$ & $0 \cdot 022$ & 0.004 & 0.022 \\
\hline $20: 3 n-9$ & $4 \cdot 6$ & 6.7 & 0.8 & $1 \cdot 6$ & 0.53 & $<0.001$ & 0.012 & 0.232 \\
\hline $18: 2 n-6$ & $5 \cdot 3$ & $3 \cdot 4$ & $5 \cdot 9$ & $3 \cdot 7$ & $0 \cdot 37$ & 0.236 & $<0.001$ & 0.686 \\
\hline $20: 4 n-6$ & $5 \cdot 8$ & $2 \cdot 4$ & $11 \cdot 2$ & $5 \cdot 6$ & 0.92 & $<0.001$ & $<0.001$ & $0 \cdot 247$ \\
\hline $22: 5 n-6$ & $1 \cdot 4$ & $2 \cdot 2$ & 1.5 & nd & 0.13 & $<0.001$ & 0.014 & 0.001 \\
\hline Total $n-6$ & $13 \cdot 4$ & 8.0 & $18 \cdot 6$ & $9 \cdot 3$ & $1 \cdot 30$ & 0.021 & $<0.001$ & $0 \cdot 149$ \\
\hline $18: 3 n-3$ & nd & nd & $3 \cdot 0$ & $0 \cdot 9$ & $0 \cdot 25$ & - & $<0.001$ & 一 \\
\hline $20: 5 n-3$ & nd & nd & $6 \cdot 3$ & $6 \cdot 8$ & 0.65 & - & 0.550 & - \\
\hline $22: 5 n-3$ & nd & nd & $4 \cdot 4$ & 1.4 & 0.41 & - & $<0.001$ & 一 \\
\hline $22: 6 n-3$ & 1.7 & nd & $19 \cdot 2$ & $13 \cdot 2$ & $0 \cdot 59$ & - & $<0.001$ & - \\
\hline Total $n-3$ & 1.7 & nd & 32.9 & $22 \cdot 3$ & 1.64 & - & $<0.001$ & - \\
\hline$P: S$ & 1.02 & 0.49 & $2 \cdot 66$ & 1.02 & $0 \cdot 25$ & $<0.001$ & $<0.001$ & 0.038 \\
\hline $20: 4 n-6 / 18: 2 n-6$ & 0.91 & 0.70 & 1.89 & 1.51 & $0 \cdot 11$ & $<0.001$ & $0-014$ & 0.45 \\
\hline $20: 3 n-9 / 20: 4 n-6$ & $0 \cdot 86$ & $2 \cdot 79$ & $0 \cdot 07$ & 0.28 & $0 \cdot 13$ & $<0.001$ & $<0.001$ & $<0.001$ \\
\hline
\end{tabular}

$\mathrm{COC}, 200 \mathrm{~g}$ casein $+50 \mathrm{~g} \mathrm{CO} / \mathrm{kg}$; COd, $20 \mathrm{~g}$ casein $+50 \mathrm{~g} \mathrm{CO} / \mathrm{kg} ; \mathrm{SAC}, 200 \mathrm{~g}$ casein $+50 \mathrm{~g} \mathrm{SA} / \mathrm{kg} ; \mathrm{SAd}, 20 \mathrm{~g}$ casein $+50 \mathrm{~g} \mathrm{SA} / \mathrm{kg}$; nd, not detected; $\mathrm{P}: \mathrm{S}$, polyunsaturated: saturated.

* For details of diets and procedures, see Table 1 and pp. 376-379.

daily food and energy intakes $/ \mathrm{kg}$ body weight (76 (SD 5) $\mathrm{g}$ and 1243 (SD 85) kJ respectively), body weights of both protein-deficient groups (SAd and COd) were only 43 and $44 \%$ of their respective control groups. Final body weights were similar in both control groups (289 (SD 9) g).

\section{Serum and lipoprotein fractions}

Protein. Protein contents of serum and total apolipoproteins of all lipoprotein fractions were significantly reduced in rats fed on low-protein diets, particularly with coconut oil (except for VLDL), compared with those fed on $200 \mathrm{~g}$ casein $/ \mathrm{kg}$ diets (Table 2).

Total cholesterol. Protein-deficient diets lowered LDL-HDL TC values in both proteindeficient groups, $\mathrm{HDL}_{2-3} \mathrm{TC}$ in the COd group and VLDL TC in the SAd group, relative to their respective control groups (Table 2). This fall concerned the two fractions (esterified and unesterified). In addition, serum and VLDL TC concentrations were depressed (essentially due to the esterified fraction) in the SAd group compared with the SAC group. Consumption of coconut oil compared with that of salmon oil enhanced the TC (esterified and unesterified) concentrations in serum and $\mathrm{HDL}_{2-3}$.

Phospholipids. VLDL PL concentrations were depressed in the COd group, and $\mathrm{HDL}_{2-3}$ PL concentrations were raised in both protein-deficient groups, compared with their respective controls (Table 2). Feeding salmon oil diets resulted in lower concentrations of serum and VLDL PL than feeding coconut oil diets.

Triacylglycerol. Serum and VLDL TG concentrations were depressed by low-protein diets relative to rats given $200 \mathrm{~g}$ casein $/ \mathrm{kg}$ diets (Table 2). Furthermore, these values were lower in rats given salmon oil diets compared with rats given coconut oil diets. 
Table 6 . The effects of control $(C)$ or protein-deficient $(d)$ diets containing coconut $(C O)$ or salmon oil $(S A)$ on major fatty acid compositions of very-low-density lipoprotein (VLDL) phospholipids ( $\mathrm{mg} / 100 \mathrm{mg}$ fatty acids) of growing rats*

(Values are means for six rats with their pooled standard errors)

\begin{tabular}{|c|c|c|c|c|c|c|c|c|}
\hline \multirow[b]{2}{*}{ Diet ... } & \multirow[b]{2}{*}{$\mathrm{COC}$} & \multirow[b]{2}{*}{ COd } & \multirow[b]{2}{*}{ SAC } & \multirow[b]{2}{*}{ SAd } & \multirow[b]{2}{*}{ SEM } & \multicolumn{3}{|c|}{$\begin{array}{c}\text { Statistical significance }(P) \\
\text { of effect of }\end{array}$} \\
\hline & & & & & & $\begin{array}{c}\text { oil type } \\
(\mathrm{CO} \text { v. SA })\end{array}$ & $\begin{array}{l}\text { level of } \\
\text { protein } \\
(\mathrm{C} v . \mathrm{d})\end{array}$ & $\begin{array}{l}\text { Interaction } \\
\text { (oil type } \times \text { level } \\
\text { of protein) }\end{array}$ \\
\hline Total saturated & 47.6 & $52 \cdot 8$ & $34 \cdot 2$ & $41 \cdot 5$ & 3.87 & 0.004 & 0.123 & 0.79 \\
\hline Total monounsaturated & $32 \cdot 1$ & $24 \cdot 3$ & $14 \cdot 7$ & $18 \cdot 3$ & $1 \cdot 29$ & $<0.001$ & 0.118 & $<0.001$ \\
\hline $20: 3 n-9$ & $5 \cdot 8$ & 8.7 & nd & nd & 0.57 & - & $<0.001$ & - \\
\hline $18: 2 n-6$ & 6.9 & $4 \cdot 3$ & 8.9 & $5 \cdot 9$ & 0.82 & 0.040 & 0.005 & 0.812 \\
\hline $20: 4 n-6$ & 49 & 3.7 & $12 \cdot 7$ & $5 \cdot 3$ & $0 \cdot 70$ & $<0.001$ & $<0.001$ & $<0.001$ \\
\hline $22: 5 n-6$ & $2 \cdot 2$ & $4 \cdot 6$ & nd & nd & 0.45 & - & $<0.001$ & - \\
\hline Total $n-6$ & $14 \cdot 0$ & $12 \cdot 6$ & $21 \cdot 6$ & $11 \cdot 2$ & 1.63 & 0.072 & 0.001 & $0 \cdot 012$ \\
\hline $18: 3 n-3$ & nd & nd & 1.0 & 0.6 & $0 \cdot 10$ & - & 0.002 & - \\
\hline $20: 5 n-3$ & nd & nd & $13 \cdot 0$ & $9 \cdot 1$ & $0 \cdot 36$ & - & $<0.001$ & - \\
\hline $22: 5 n-3$ & nd & nd & $3 \cdot 7$ & $1 \cdot 3$ & 0.45 & - & $<0.001$ & - \\
\hline $22: 6 n-3$ & nd & nd & $11 \cdot 2$ & $15 \cdot 8$ & 0.95 & - & $<0.001$ & - \\
\hline Total $n-3$ & nd & nd & 28.9 & $26 \cdot 8$ & $2 \cdot 25$ & - & 0.380 & - \\
\hline$P: S$ & 1.09 & $0 \cdot 86$ & 1.90 & 1.35 & $0 \cdot 14$ & $<0.001$ & 0.011 & $0 \cdot 267$ \\
\hline $20: 4 n-6 / 18: 2 n-6$ & 0.71 & 0.86 & 1.42 & 0.89 & 0.09 & $<0.001$ & 0.047 & 0.001 \\
\hline $20: 3 n-9 / 20: 4 n-6$ & $1 \cdot 18$ & $2 \cdot 35$ & - & - & $0 \cdot 22$ & - & $<0.001$ & - \\
\hline
\end{tabular}

COC, $200 \mathrm{~g}$ casein $+50 \mathrm{~g} \mathrm{CO} / \mathrm{kg} ; \mathrm{COd}, 20 \mathrm{~g}$ casein $+50 \mathrm{~g} \mathrm{CO} / \mathrm{kg} ; \mathrm{SAC}, 200 \mathrm{~g}$ casein $+50 \mathrm{~g} \mathrm{SA} / \mathrm{kg} ; \mathrm{SAd}, 20 \mathrm{~g}$ casein $+50 \mathrm{~g} \mathrm{SA} / \mathrm{kg}$; nd, not detected; P:S, polyunsaturated: saturated.

* For details of diets and procedures, see Table 1 and pp. 376-379.

Fatty acid composition ( $\mathrm{g} / 100 \mathrm{~g}$ total fatty acids) of serum and lipoproteins

Serum and VLDL triacylglycerols. Protein-deficient diets diminished the polyunsaturated : saturated fatty acid (P:S) value in serum and VLDL TG (Tables 3 and 4). The fatty acid 22:5n-6 was not detected in VLDL TG of the SAd group but was raised in serum of both protein-deficient groups. The $20: 4 n-6: 18: 2 n-6$ value in serum and the $20: 3 n-9: 20: 4 n-6$ value (Holman's (1960) EFA deficiency index) in VLDL were enhanced by protein-deficient diets. In addition, the COd group, relative to the COC group, had lower linoleic and arachidonic acids, and thereby total $n-6$ in both serum and VLDL, whereas the SAd group, relative to the SAC group, had lower $20: 5 n-3$ and total $n-3$ fatty acids in serum and VLDL, but enhanced 20:4n-6 and total $n-6$ fatty acids in serum, and 22:6n-3 in VLDL.

Compared with coconut oil diets, salmon oil diets raised total $n$-3 fatty acids, the $20: 3 n$ $9: 20: 4 n-6$ and $P: S$ values, whereas they diminished total saturated, monounsaturated, $20: 4 n-6$ and total $n-6$ fatty acids in serum and VLDL TG fractions. In addition, rats fed on salmon oil diets relative to those fed on coconut oil diets had higher $20: 4 n-6: 18: 2 n-6$ ratios in VLDL TG, unlike the levels obtained in serum TG. Furthermore $n-3$ fatty acids were generally not detected with coconut oil diets.

Serum, VLDL and $H D L_{2-3}$ phospholipids. Low-protein diets enhanced total saturated and the $20: 3 n-9: 20: 4 n-6$ value and diminished $18: 2 n-6,20: 4 n-6$, total $n-6$ fatty acids and the $\mathrm{P}: \mathrm{S}$ value in serum and VLDL PL fractions (Tables 5 and 6). In addition, in the COd group, relative to the COC group, 22:5n-6 was generally higher in overall PL studied (serum, VLDL, $\mathrm{HDL}_{2-3}$ ) whereas, in the SAd group, relative to the SAC group, 22:6n-3 was enhanced in VLDL- and $\mathrm{HDL}_{2-3}-\mathrm{PL}$ (Tables 5-7). Compared with salmon oil diets, coconut oil diets increased total saturated, monounsaturated, $20: 3 n-9$ and the $20: 3 n$ - 
Table 7. The effects of control $(C)$ or protein-deficient $(d)$ diets containing coconut $(C O)$ or salmon oil $(S A)$ on major fatty acid compositions of high-density lipoprotein $(H D L)$ phospholipids $(\mathrm{mg} / 100 \mathrm{mg}$ fatty acids) of growing rats*

(Values are means for six rats with their standard errors)

\begin{tabular}{|c|c|c|c|c|c|c|c|c|}
\hline \multirow[b]{2}{*}{ Diet... } & \multirow[b]{2}{*}{$\mathrm{COC}$} & \multirow[b]{2}{*}{ COd } & \multirow[b]{2}{*}{ SAC } & \multirow[b]{2}{*}{ SAd } & \multirow[b]{2}{*}{ SEM } & \multicolumn{3}{|c|}{$\begin{array}{c}\text { Statistical significance }(P) \\
\text { of effect of }\end{array}$} \\
\hline & & & & & & $\begin{array}{c}\text { oil type } \\
\text { (CO v. SA) }\end{array}$ & $\begin{array}{l}\text { level of } \\
\text { protein } \\
(\mathrm{C} v . \mathrm{d})\end{array}$ & $\begin{array}{c}\text { Interaction } \\
\text { (oil type } \times \text { level } \\
\text { of protein) }\end{array}$ \\
\hline Total saturated & $48 \cdot 0$ & $58 \cdot 5$ & $32 \cdot 3$ & $39 \cdot 1$ & 3.53 & $<0.001$ & 0.023 & 0.608 \\
\hline Total monounsaturated & $31 \cdot 1$ & $19 \cdot 9$ & $20 \cdot 9$ & $17 \cdot 5$ & 1.59 & $<0.001$ & $<0.001$ & 0.023 \\
\hline $20: 3 n-9$ & $10 \cdot 1$ & $12 \cdot 4$ & $\operatorname{tr}$ & $\operatorname{tr}$ & $1 \cdot 12$ & - & $0 \cdot 067$ & - \\
\hline $18: 2 n-6$ & $3 \cdot 5$ & $1 \cdot 7$ & $6 \cdot 5$ & $5 \cdot 3$ & 0.56 & $<0.001$ & 0.014 & 0.601 \\
\hline $20: 4 n-6$ & $4 \cdot 3$ & $3 \cdot 3$ & $13 \cdot 0$ & $15 \cdot 3$ & 0.73 & $<0.001$ & $0 \cdot 384$ & 0.035 \\
\hline $22: 5 n-6$ & $2 \cdot 2$ & $3 \cdot 8$ & nd & nd & $0 \cdot 20$ & - & $<0.001$ & - \\
\hline Total $n-6$ & 10.0 & $8 \cdot 8$ & $19 \cdot 5$ & $20 \cdot 6$ & 1.35 & $<0.001$ & $>0.90$ & 0.405 \\
\hline $18: 3 n-3$ & nd & nd & $2 \cdot 0$ & $\operatorname{tr}$ & - & - & - & - \\
\hline $20: 5 n-3$ & nd & nd & $12 \cdot 5$ & $7 \cdot 8$ & $0 \cdot 29$ & - & $<0.001$ & - \\
\hline $22: 5 n-3$ & nd & nd & $2 \cdot 7$ & 2.9 & $0 \cdot 25$ & - & 0.420 & - \\
\hline $22: 6 n-3$ & nd & nd & $9 \cdot 2$ & $11 \cdot 6$ & 0.51 & - & $<0.001$ & $\ldots$ \\
\hline Total $n-3$ & nd & nd & 26.4 & $22 \cdot 3$ & 1.05 & - & 0.003 & - \\
\hline $\mathbf{P}: \mathbf{S}$ & 1.06 & $0 \cdot 70$ & 2.06 & $1 \cdot 54$ & $0 \cdot 21$ & $<0.001$ & 0.048 & 0.708 \\
\hline $20: 4 n-6 / 18: 2 n-6$ & $1 \cdot 22$ & 1.94 & $2 \cdot 00$ & $2 \cdot 88$ & $0 \cdot 22$ & $<0.001$ & 0.001 & 0.722 \\
\hline $20: 3 n-9 / 20: 4 n-6$ & $2 \cdot 34$ & $3 \cdot 75$ & - & - & 0.55 & - & 0.028 & - \\
\hline
\end{tabular}

COC, $200 \mathrm{~g}$ casein $+50 \mathrm{~g} \mathrm{CO} / \mathrm{kg} ; \mathrm{COd}, 20 \mathrm{~g}$ casein $+50 \mathrm{~g} \mathrm{CO} / \mathrm{kg} ; \mathrm{SAC}, 200 \mathrm{~g}$ casein $+50 \mathrm{~g} \mathrm{SA} / \mathrm{kg} ; \mathrm{SAd}, 20 \mathrm{~g}$ casein $+50 \mathrm{~g} \mathrm{SA} / \mathrm{kg}$; nd, not detected; tr, trace $(<0 \cdot 1) ; \mathrm{P}: \mathrm{S}$, polyunsaturated : saturated.

* For details of diets and procedures, see Table 1 and pp. 376-379.

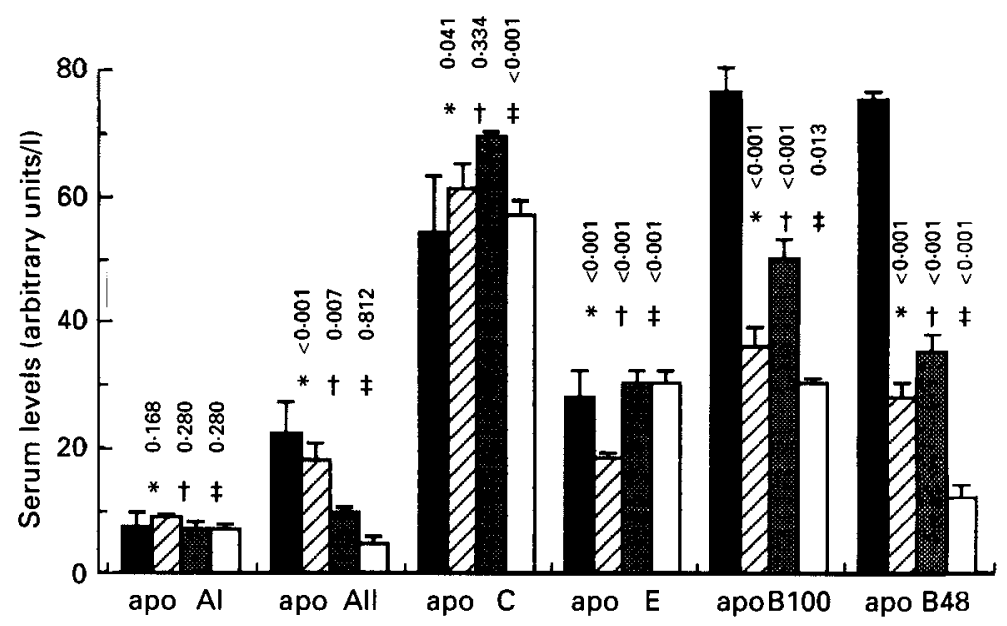

Fig. 1. Very-low-density lipoprotein (VLDL)-apolipoprotein (apo) distribution in young rats fed on proteindeficient diets with coconut or salmon oils for $28 \mathrm{~d}$. Values are means with their standard errors represented by vertical bars for six rats. $(\square), 200 \mathrm{~g}$ casein $+50 \mathrm{~g}$ coconut oil $/ \mathrm{kg} ;(\square), 20 \mathrm{~g}$ casein $+50 \mathrm{~g}$ coconut oil $/ \mathrm{kg}$; $(B)$, $200 \mathrm{~g}$ casein $+50 \mathrm{~g}$ salmon oil $/ \mathrm{kg} ;(\square), 20 \mathrm{~g}$ casein $+50 \mathrm{~g}$ salmon oil $/ \mathrm{kg}$. Statistical significance $(P)$ is given for the effects of: * oil type (coconut $v$. salmon), $\dagger$ level of protein $(200 \mathrm{~g} \mathrm{v.} 20 \mathrm{~g})$, $\ddagger$ interaction (oil type $\times$ level of protein). 


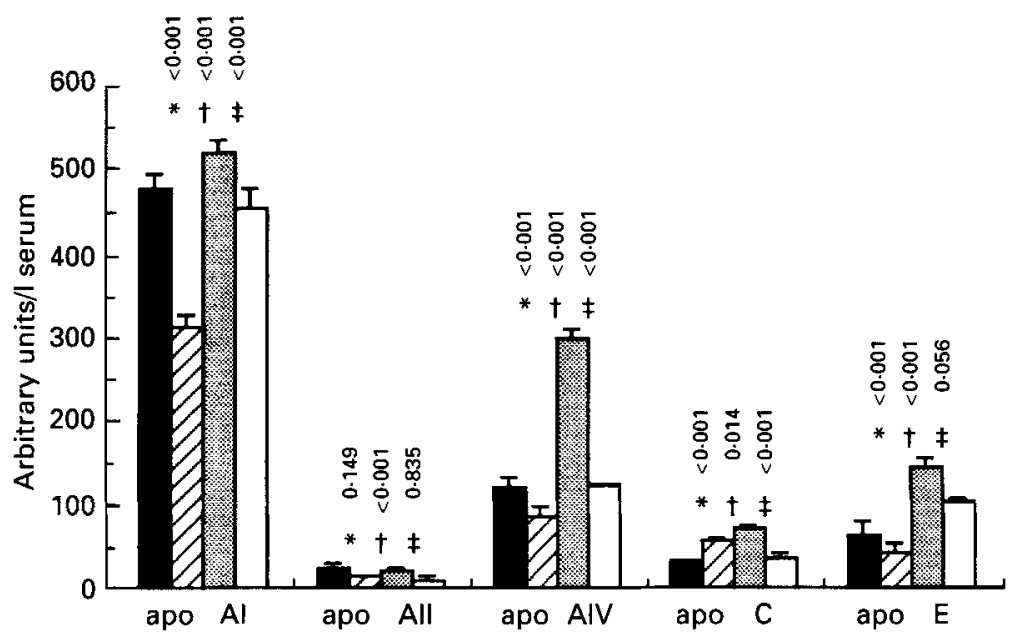

Fig. 2. High-density lipoprotein ${ }_{2-3}\left(\mathrm{HDL}_{2-3}\right)$-apolipoprotein (apo) distribution in young rats fed on proteindeficient diets with coconut or salmon oils for $28 \mathrm{~d}$. Values are means with their standard errors represented by vertical bars for six rats. (D), $200 \mathrm{~g}$ casein $+50 \mathrm{~g}$ coconut oil $/ \mathrm{kg} ;(\square), 20 \mathrm{~g}$ casein $+50 \mathrm{~g}$ coconut oil $/ \mathrm{kg} ;($ (日) $), 200 \mathrm{~g}$ casein $+50 \mathrm{~g}$ salmon oil $/ \mathrm{kg} ;(\square), 20 \mathrm{~g}$ casein $+50 \mathrm{~g}$ salmon oil $/ \mathrm{kg}$. Statistical significance $(P)$ is given for the effects of: * oil type (coconut $v$. salmon), $\dagger$ level of protein $(200 \mathrm{~g} \mathrm{v.} 20 \mathrm{~g})$, $\ddagger$ interaction (oil type $\times$ level of protein).

$9: 20: 4 n-6$ value but decreased $20: 4 n-6$, total $n-6$ and $n-3$ fatty acids, the $\mathrm{P}: \mathrm{S}$ and the $20: 4 n-$ $6: 18: 2 n-6$ values in overall PL (serum, VLDL, $\mathrm{HDL}_{2-3}$ ).

\section{$V L D L$-apolipoprotein composition}

Apo $B_{100}$, apo $B_{48}$ and apo $A_{I V}$ were depressed in rats fed on low-protein diets (Fig. 1). Compared with their respective controls, the COd group had lower apo $\mathrm{E}$ and the SAd group lower apo $\mathrm{C}$. Apo $\mathrm{A}_{\mathrm{I}}$ was not affected by protein-deficient diets. Salmon oil diets resulted in lower apo $B_{100}$, apo $B_{48}$ and apo $A_{I V}$ and higher apo $C$ values than those obtained with coconut oil diets.

\section{$H D L_{2-3}$-apolipoprotein composition}

Total apolipoproteins of $\mathrm{HDL}_{2-3}$ were higher in the SAC group compared with those of the COC group (Fig. 2). Protein-deficient diets depressed these values in both protein deficient groups. However, compared with their respective controls, apo $\mathrm{A}_{I I}$ in the SAd group and apo $\mathrm{E}$ and apo $\mathrm{C}$ in the $\mathrm{COd}$ group were not affected by protein deficiency. Furthermore, in the SAd group, apo $A_{\mathrm{IV}}, \mathrm{C}$ and $\mathrm{E}$ values were generally depressed and apo $\mathrm{A}_{1}$ was less affected, compared with those in the COd group.

\section{DISCUSSION}

Fatty liver occurs in children suffering from kwashiorkor. This liver lipid accumulation (mainly TG) is attributed by Flores et al. (1970) and Meghelli-Bouchenak et al. (1989 b) to the defect of hepatic TG exportation by VLDL. The presence of fatty liver and similarity between the skin lesions of kwashiorkor and those described in experimental EFA deficiency lead to the hypothesis that protein and EFA deficiencies may both be involved in chronic malnutrition. Our results demonstrate a similar association between protein deficiency and fatty liver in a rat model.

Protein deficiency has a strong influence on the PUFA pattern of heart, liver and serum (Hill \& Holman, 1980). PUFA are mainly rapidly incorporated into structural lipids or stored in adipose tissues and are not very useful for oxidation. The Triene:tetraene ratio, 20:3n-9:20:4n-6 (limit of normality $<0 \cdot 2$ ), introduced by Holman (1960) to assess EFA 
deficiency was too simplistic, and serum PUFA profile, which reveals disproportion between $n-6, n-3$, and $n-9$ PUFA, is preferred as an indicator of EFA status. In the present work, to compare dietary variables, data are only considered with respect to proportions (relative percentages by weight) and, therefore, do not provide actual amounts of fatty acids. The reason for this is that the properties of cell membranes are influenced by the proportions of various types of fatty acids in their lipids, and because serum PL reflect the EFA status of tissue lipids (Holman, 1986).

In our study protein malnutrition decreased the protein content of serum and lipoproteins, yet the values obtained with salmon oil diets were higher than those obtained with hydrogenated coconut oil diets, except for VLDL (due to apo $\mathrm{B}_{100}$ and apo $\mathrm{B}_{48}$ ). Serum and VLDL TG contents were lowered with protein-deficient diets but more when salmon oil was associated. These results for proteins and TG are consistent with those in the literature, but those for TC and PL are inconsistent. In our study the PL contents of VLDL were diminished but those of $\mathrm{HDL}_{2-3}$ were enhanced in rats fed on low-protein diets. These rats had a lower LDL- $\mathrm{HDL}_{1}$ TC content. Furthermore, in the SAd group, serum and VLDL TC, and in the COd group $\mathrm{HDL}_{2-3} \mathrm{TC}$ were lower than in the SAC and COC groups respectively. Koleztko et al. (1986) have reported that total amounts of PUFA as well as the $\mathrm{P}: \mathrm{S}$ value were severely reduced in plasma PL and cholesteryl esters in malnourished Nigerian children. In the overall lipid fractions studied we also found a decreased $P: S$ value with protein-deficient diets, but the values in the presence of salmon oil were higher than those obtained with hydrogenated coconut oil. Similarly, Holman's (1960) index was enhanced for both protein-deficient groups in VLDL TG but only with hydrogenated coconut oil in overall PL. Koleztko et al. (1986) reported an elevated $20: 3 n$ 9:20:4n-6 value in the serum TG fraction, but not in PL and cholesteryl esters in malnourished children.

Fatty acid profiles of serum PL showed a fall in linoleic, linolenic and arachidonic acids and consequently depressed total $n-6$ and $n-3$ fatty acids with both protein-deficient diets. In VLDL and $\mathrm{HDL}_{2-3} \mathrm{PL}$ fractions, the same results were observed in the COd group, while in the SAd group, linoleic, arachidonic and total $n-6$ fatty acids were not reduced. These changes showed that the $20: 4 n-6: 18: 2 n-6$ ratio is not the best reflection of $\Delta 6$ desaturase activities. Mercuri et al. (1979) and De Tomas et al. (1980) have shown that partial protein deprivation is associated with decreased arachidonate:linoleate values in PL from rat liver and have postulated that 18:2n-6 accumulation was due to impaired chain elongation or desaturation or both in protein-deficient rats. However, Gerson \& Wong (1978) showed that the incubation in vitro of liver microsomes of rats fed on a protein-free diet for 7 weeks revealed unchanged chain elongation and desaturation enzyme activities. Narce et al. (1988) showed that hepatic microsomal $\Delta 6$ - and $\Delta 5$-desaturase activities are not strictly paralleled by changes in the altered fatty acid composition of liver total lipids.

It is well known that $18: 3 n-3$, as $20: 5 n-3$ (Garg et al. 1988), competes with $18: 2 n-6$ and 18: $1 n-9$ for $\Delta 6$ desaturase. The competitive activities of the three substrates for $\Delta 6$ desaturation are $n-3>n-6>n-9$. In the present study the arachidonate:linoleate value, though subject to a wide variation, showed, for example, increased values in the COd group (except for serum PL), yet we did not find 18:2n-6 accumulation in protein-deficient groups as observed by De Tomas et al. (1980). The variations in this ratio with protein deficiency were the result of both arachidonic and linoleic acid decreases, but for each fatty acid the fall was not the same. On the other hand, when $18: 2 n-6$ was diminished by protein-deficient diets it was accompanied by a rise in $20: 3 n-9$ (particularly in the COd group and in serum PL of the SAd group), or in total monounsaturated fatty acids (in VLDL PL of the SAd group), even though salmon oil supplies a little of both arachidonic and linoleic acids $(1 \cdot 1$ and $4.0 \mathrm{~g} / 100 \mathrm{~g}$ respectively). For these reasons we conclude that $\Delta 6$ and $\Delta 5$ desaturase activities may be reduced only a little in our protein-deficient rats. In support of this view, 
we have shown in a previous study with the same experimental protocol that there was no correlation between microsomal desaturation rate and microsome PL profiles, and that the PUFA incorporated into microsomal membranes have no effect on desaturases (Ulmann et al. 1992).

Fatty acid profiles of VLDL and $\mathrm{HDL}_{2-3} \mathrm{PL}$ fractions showed a greater proportion of 22:5n-6 and 22:6n-3 in the COd and SAd groups respectively. These increments could be attributable to higher hepatic microsomal $\Delta 4$ desaturase activity or to a lower utilization of these fatty acids by other tissues ( $\Delta 4$ desaturase activity involves the desaturation of $22: 5 n-3$ to $22: 6 n-3$ or $22: 4 n-6$ to $22: 5 n-6$ ). This activity increases with $n-3$ fatty acid deficiency in rat liver (Hagve et al. 1984).

Total VLDL apolipoproteins are reduced by protein deficiency, probably on account of a decrease in synthesis as observed by Yagasaki \& Kametaka (1984) and MeghelliBouchenak et al. (1987). Results obtained in previous reports of rats subjected to $20 \mathrm{~g}$ casein or $50 \mathrm{~g}$ gluten and $50 \mathrm{~g}$ sunflower oil $/ \mathrm{kg}$ diet showed that the reduced synthesis of liver VLDL apolipoproteins, indeed apo $\mathrm{B}_{100}$, apo $\mathrm{B}_{48}$ and apo $\mathrm{C}$ which are the main apolipoproteins synthesized by liver in rats, is a cause of impaired hepatic TG export, which results in fatty liver (Meghelli-Bouchenak et al. 1989 b). The fall in total apolipoproteins of VLDL and $\mathrm{HDL}_{2-3}$ induced by protein deficiency in this present study was less pronounced (and mainly due to apo $\mathbf{B}_{100}$ and apo $\mathrm{B}_{48}$ ) than in previous work (Bouziane et al. 1992). Furthermore, we have shown in a previous report that protein deficiency reduced the halflives of VLDL apolipoproteins and increased their clearance from VLDL, probably owing to an increase in peripheral uptake of different organs but not to a higher uptake by liver (Meghelli-Bouchenak et al. 1991). Apo $B_{100}$ and apo $B_{48}$ in both protein-deficient groups, apo $A_{I}$ and apo $E$ in the COd group and apo $A_{I V}$ in the SAd group were the most affected by protein deficiency. With salmon oil diets, apo $C$, apo $A_{I}$, apo $E$ and apo $A_{I V}$ values were greater than those obtained with coconut oil diets.

In conclusion, giving protein-deficient diets to rats for a relatively short period of $28 \mathrm{~d}$ led to a reduced $\mathrm{P}: \mathrm{S}$ ratio in serum phospholipids even in the presence of apparently adequate levels of dietary PUFA, suggesting an impairment in 'availability' of PUFA. One consequence may be a modification of membrane viscosity and the functions of integral proteins (Léger et al. 1987), while another could be decreased availability of eicosanoids, since arachidonic and eicosapentaenoic acids are precursors of these important metabolic regulators. This may provide an explanation for the link between protein malnutrition and EFA deficiency with their common clinical features of loss of hair, dry scaly dermatitis, tendency to superinfection and increased water permeability. It may be prudent to include adequate amounts of EFA in the rehabilitation diets of infants suffering from kwashiorkor.

The authors thank Anne Magnet, a linguist at the University of Burgundy, for editing the manuscript in English. This work was supported by research grants 86 MES 25 from the French Foreign Office and a financial support from the Regional Council of Burgundy.

\section{REFERENCES}

Bartlett, G. R. (1958). Phosphorus assay in column chromatography. Journal of Biological Chemistry 234, 466-468.

Bouziane, M., Prost, J. \& Belleville, J. (1992). Changes in serum and lipoprotein fatty acids of growing rats fed protein-deficient diets with low or adequate linolenic acid concentrations. Journal of Nutrition 122, $2037-2046$.

Council of European Communities (1986). Council instructions about the protection of living animals used in scientific investigations. Official Journal of European Communities (J. O. 86/609/CEE) L 358, 1-28.

De Tomas, M. E., Mercuri, O. \& Rodrigo, A. (1980). Effects of dietary protein and EFA deficiency on liver $\Delta 5$, $\Delta 6$ and $\Delta 9$ desaturase activities in the early developing rat. Journal of Nutrition 110, 314-319.

Dhansay, M. A., Benade, A. J. S. \& Donald, P. R. (1991). Plasma lecithin-cholesterol acyltransferase activity and plasma lipoprotein composition and concentrations in kwashiorkor. American Journal of Clinical Nutrition $\mathbf{5 3}$, $512-519$. 
Flores, H., Pak, N., Maccioni, A. \& Monckeberg, F. (1970). Lipid transport in kwashiorkor. British Journal of Nutrition 24, 1005-1011.

Folch, J., Lees, M. \& Sloane Stanley, G. H. (1957). A simple method for the isolation and purification of total lipids from animal tissues. Journal of Biological Chemistry 226, 497-509.

Gambert, P., Lallemand, C. \& Archambault, A. (1979). Assessment of serum cholesterol by two methods: gas liquid chromatography on a capillary column and chemical ionization-mass fragmentography with isotopic dilutions of $\left[3,4^{13} \mathrm{C}\right]$ cholesterol as internal standard. Journal of Chromatography 162, 1-6.

Gambert, P., Lallemand, C., Athias, A. \& Padieu, P. (1982). Alterations of HDL-cholesterol distribution induced by incubation of human serum. Biochimica et Biophysica Acta 713, 1-9.

Garg, M. L., Sebokova, E., Thomson, A. B. R. \& Clandinin, M. T. (1988). $\Delta 6$ desaturase activity in liver microsomes of rats fed diets enriched with cholesterol and/or w3 fatty acids. Biochemical Journal 249, 351-356.

Gerson, T. \& Wong, H. N. (1978). The effect of protein deficiency on some rat liver metabolic enzymes and coenzyme A. Lipids 13, 446-450.

Hagve, T. A. \& Christophersen, B. O. (1984). Effect of dietary fats on arachidonic acid and eicosapentaenoic acid biosynthesis and conversion to $\mathrm{C}_{22}$ fatty acids in isolated rat liver cells. Biochimica et Biophysica Acta 796, $205-217$.

Harris, W., Connor, W.\& McMurry, M. (1983). The comparative reductions of the plasma lipids and lipoproteins by dietary polyunsaturated fats: salmon oil versus vegetable oils. Metabolism 32, 179-184.

Havel, R. J., Eder, H. A. \& Bragdon, J. H. (1955). The distribution and chemical composition of ultracentrifugally separated lipoproteins in human serum. Journal of Clinical Investigations 34, 1345-1363.

Hill, E. G. \& Holman, R. T. (1980). Effect of dietary protein level upon essential fatty acid (EFA) deficiency. Journal of Nutrition 110, 1057-1060.

Holman, R. T. (1960). The ratio of trienoic:tetraenoic acids in tissue lipids as a measure of essential fatty acid requirement. Journal of Nutrition 70, 405-411.

Holman, R. T. (1986). Nutritional and functional requirements for essential fatty acids. Progress in Clinical and Biological Research 222, 211-228.

Hornstra, G. (1989). Influence of dietary fish oil on arterial thrombosis and atherosclerosis in animal models and in man. Journal of Internal Medicine 225, 53-60.

Hostmark, A. T., Spydevold, O. \& Lystad, E. (1982). Plasma high density lipoprotein sub-group distribution in rats fed diets with varying amounts of sucrose and sunflower oil. Lipids 17, 489-499.

Koletzko, B., Abiodun, P. O., Laryea, M. D. \& Bremer, H. J. (1986). Fatty acid composition of plasma lipids in Nigerian children with protein-energy malnutrition. European Journal of Pediatrics 145, 109-115.

Léger, C., Fremont, L., Alessandrini, J. M., Christon, R. \& Linard, D. (1987). Les acides gras essentiels ont-ils une fonction structuro-modulatrice membranaire spécifique? (Have essential fatty acids a specific structuralmodulating membrane function?) Cahier de Nutrition et de Diététique XXII, 2, 159-165.

Lowry, O. H., Rosebrough, N. J., Farr, A. L. \& Randall, R. I. (1951). Protein measurement with Folin phenol reagent. Journal of Biological Chemistry 193, 265-275.

Meghelli-Bouchenak, M., Belleville, J. \& Boquillon, M. (1989a). Hepatic steatosis and serum very low density lipoproteins during two types of protein malnutrition followed by balanced refeeding. Nutrition 5, 321-329.

Meghelli-Bouchenak, M., Boquillon, M. \& Belleville, J. (1987). Time course of changes in rat serum apolipoproteins during consumption of different low protein diets followed by a balanced diet. Journal of Nutrition 117, 641-649.

Meghelli-Bouchenak, M., Boquillon, M. \& Belleville, J. (1989b). Serum lipoprotein composition and amounts during the consumption of two different low protein diets followed by a balanced diet. Nutrition Reports International 39, 323-343.

Meghelli-Bouchenak, M., Boquillon, M., Belleville, J. \& Prost, J. (1991). Metabolism of serum VLDL-, LDL-, and HDL-apoproteins in rats on two different low protein diets. Nutrition Research 11, 575-586.

Mercuri, O., De Tomas, M. E. \& Itarte, H. (1979). Prenatal protein depletion and $\Delta 9, \Delta 6$ and $\Delta 5$ desaturases in the rat. Lipids 14, 822-825.

Narce, M., Poisson, J. P., Belleville, J. \& Chanussot, B. (1988). Time-course effects of protein malnutrition on hepatic fatty acids $\Delta 6$ and $\Delta 5$ desaturation in the growing rat. British Journal of Nutrition $60,389-402$.

Redgrave, T. G., Roberts, D. C. K. \& West, C. E. (1975). Separation of plasma lipoprotein by density gradient ultracentrifugation. Analytical Biochemistry 65, 42-49.

Slover, H. T. \& Lanza, E. (1979). Quantitative analysis of food fatty acids by capillary gas chromatography. Journal of the American Oil Chemists' Society 56, 933-943.

Stahl, E., Schröter, G., Kraft, G. \& Renz, E. (1956). Thin layer chromatography (the method affecting factors, and a few examples of application). Pharmazie 11, 633-637.

Ulmann, L., Bouziane, M., Mimouni, M., Belleville, J. \& Poisson, J. P. (1992). Relationship between rat liver microsomal $\Delta 6$ and $\Delta 5$ desaturase activities and fatty acid composition: comparative effects of coconut and salmon oils during protein restriction. Journal of Nutritional Biochemistry 3, 188-193.

Yagasaki, K. \& Kametaka, M. (1984). Apolipoprotein synthesis by the fatty liver of rats fed a low whole egg protein diet. Nutrition Reports International 29, 533-540. 